\title{
An Integer Programming Model for Gate Assignment Problem at Airline Terminals
}

\author{
Chong Kok Chun ${ }^{\mathrm{a}}$, Syarifah Zyurina Nordin ${ }^{\mathrm{b}, *}$ \\ ${ }^{a, b}$ Department of Mathematical Science, Faculty of Science, Universiti Teknologi Malaysia, 81310 UTM Johor Bahru, Johor, Malaysia. \\ *Corresponding Author: szyurina@utm.my
}

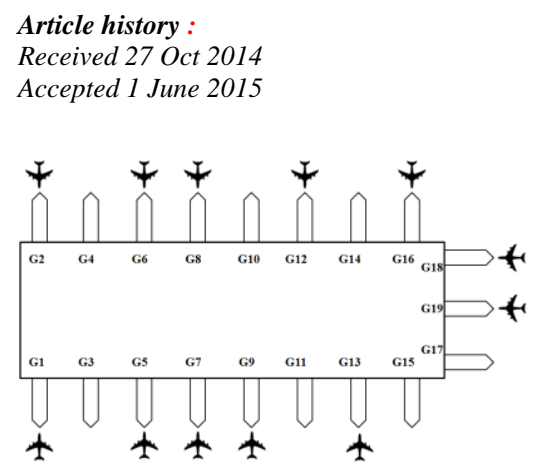

Article history :

Received 27 Oct 2014

\section{INTRODUCTION}

In daily airline's operation, Gate Assignment Problem (GAP) plays an important role for schedules an arriving flight to a certain gate. The research in [1] mentioned that there are some of the factors that has to be considered for schedule of the planes arrive and depart at the airline terminal such as passenger walking distances, baggage transfer, ramp congestion, aircraft size, aircraft rotation, and aircraft service requirement. Besides, the delay of the flights will be happened that linked to the weather conditions or the events of other airports.

Since an arriving plane remains at a gate, the due date of scheduled departure time can be viewed and the performance of airplane is measured accordingly. Meanwhile, these statements stated in [2] which are arriving passengers have to be deplaned, the plane has to be serviced, and departing passengers have to be boarded within the time.

In the airline terminal, the hub-and spoke system has occurred in a large amount of baggage and the flow of the passengers travelling between the flights. In the other word, the passengers required to walk from the arriving gate to the next departure gates for their journey and claim the baggage at the baggage claim area. Therefore, the baggage handlings process needed to be done before the departure time for the next flight which stated in the research [3].

In this paper, we have to consider the hub-andspoke system that suitable to describe the flow of the GAP.
These models can be formulated as zero-one integer problem, which obtain optimal solution for GAP that assigns the flights to the suitable gates. There are two considerations: a) walking distance of the passengers between the gates (arrival to departure) and b) the distance of baggage transport from arrival gate to the next destinations (departure gates). As well, these considerations are the objective functions. Therefore, the calculated total distance is based on these two considerations. Usually, there are two classes of constraints are required in the modeling: i) every flight only been assigned to one gate and ii) no two airplanes will be assigned at the same time as the same gate.

Next, the objective function is the minimization of the total distance as well as to assign the aircraft to a suitable gate. In the research [4], it mentioned that total distance divided into two parts which are the total walking distance of the passenger inside the terminal to reach their departure gate and the total distance of the baggage transport flow in the apron. On findings the optimal solution for this model, there did not take the parts of the delay of the flight. There have three cases needed to be tested with the ratio of the weighted of total walking distances to weighted of the total distances of baggage transport: a) ratio of $3: 7$, b) ratio of $5: 5$, and c) ratio of $7: 3$. Thus, the total distance of the two criterions was the important for the gate assignment. 


\section{LITERATURE REVIEWS}

GAP plays an important role in schedule the flights to a certain gates. Without GAP, accident might occur when there are two airplanes assigned in the same gate since both aircrafts will land on the same runway. The problem has specific levels need to be handled for finding a suitable gate to assign. These levels are the capacity of the gates that provide accommodation for the flights and development of the daily plan before the actual day of operation. Furthermore, the irregular conditions such as failure of the mechanical, bad weather and the delays of the flight also the levels usually to be handled, which stated in [1].

Hub-and- spoke system commonly use in the airline operation. Airlines choose flight schedules and route prices to maximize profit, considering competitors' decisions which stated in [5]. Besides, the system can be use in the transportation, management, financial problem and other applications in real life problem. In this study, hub-and-spoke operates in the schedule an arriving flight to a gate. There are considerations need to be observed for the distance of the passengers that need to depart the next gate and the baggage transport distance from the arrival gate to the departure gate. For those considerations, it undergoes the hub-and spoke system that reduce the both distances. In the research [3], it mentioned that with these considerations, the optimal performance will be well perform so that the flight is undergoes the optimal of expectation without late due-date

The integer linear programming is the mathematical programming problems in which all variables are constrained to integer values which stated in [6]. The integer constraints are involved in many engineering, industrial and financial applications. The hub-and-spoke system in the gate assignment problem is modeled as ILP model with the binary decision with zero-one problem. In the study, the zero-one problem is suitable for the problem that assigns a flight to a gate. The reason is all the variables are restricted to have only the value of zero or one.

Zero-one problem is the problem that required to take the zero or one value for all the variables. Usually, the variables have an abstract interpretation which simply indicate some of the activity or some of the particular matching or assignment takes place whether occurs or not which stated in [7]. Many scheduling problems can be formulated using zero-one variables. For example, in examination timetabling, we define the variable $x_{i j}$ with a value of 1 if examination $i$ is assigned to the period $j$.

$$
x_{i j}= \begin{cases}1, & \text { if examination } i \text { is assigned toperiod } j \\ 0, & \text { otherwise }\end{cases}
$$

Branch-and-bound can be considered the most common methods for solving ILP. It just the way for takes a hard problem and splits it into two or more easier sub-problems.
The sub-problems will split again since the sub-problems are too hard which mentioned in [5].

\section{MATHEMATICAL MODEL}

Figure In this section, we provide a mathematical model for gate assignment which attempts to assign the flights to the gates to minimize the total distance which consists of two objectives: a) the total of passenger traveling distance and b) the total baggage transport distance.

The following notations are used in this GAP problem:

$$
\begin{array}{ll}
\text { Indices: } \\
i & \text { Index for arriving flights } \\
j & \text { Index for gates } \\
k & \text { Index for departure gates }
\end{array}
$$

\section{Parameter:}

$p_{i, k} \quad$ Number of passengers that arriving on flight $i$ and departing from gate $k$.

$d p_{k, j} \quad$ Unit distance (in yards) for passengers from gate $k$ to gate $j$.

$T P_{i, j} \quad$ Total walking distance for all passengers on flight $i$ assigned to arrival gate $j$.

$t_{i, k} \quad$ Number of trips to transport baggage from flight $I$ to departing gate $k$.

$d b_{k, j} \quad$ Unit distance (in yards) to transport baggage on ramp from departing gate $k$ to arriving gate $j$.

$T B_{i, j} \quad$ Total transport distance for all baggage on flight $i$ assigned to arrival gate $j$.

$w_{1} \quad$ Weight assigned to total passenger walking distances.

$w_{2} \quad$ Weight assigned to total baggage transport distances.

$T P_{i, j}$ and $T B_{i, j}$ can be calculated as follows

$T P_{i, j}=\sum_{k \in K} p_{i, k} \cdot d p_{k, j} \quad$ for all $i \in F$ and $j \in G$

$T B_{i, j}=\sum_{k \in K} t_{i, k} \cdot d b_{k, j} \quad$ for all $i \in F$ and $j \in G$

Variables

$x_{i j}= \begin{cases}1 & \text { if flight } i \text { is assigned to gate } j \\ 0 & \text { otherwise }\end{cases}$

Model:

Minimize 


$$
\sum_{i \in F} \sum_{j \in G}\left(w_{1} T P_{i, j} x_{i, j}+w_{2} T B_{i, j} x_{i, j}\right)
$$

subject to

$$
\begin{array}{ll}
\sum_{j \in G} x_{i, j}=1 & \forall i \\
\sum_{i \in F} x_{i j}=1 & \forall j \\
x_{i j}=0 \text { or } 1 & \forall(i, j)
\end{array}
$$

Objective (1) is the total distance which accommodates for both passenger and baggage traveling and transport distances. Constraint (2) ensure that every flight must be assigned to one gate only and constraint (3) requires that the gates only available to one flight to arrive. Constraint (4) is alternative of the flights either assigned to the gates.

\section{EXPERIMENTAL DESIGN}

We implement the ILP model on a personal computer with the simulation data for the GAP at San Francisco Airport specifically at C Concourse [1]. There are 19 gates as shown in Figure 1. From the figure, there are 12 aircrafts already at the gates for their departures in the airport. At the same time, 7 aircrafts will be arriving to the airport and will be assigned to the 7 remaining gates (ie. Gate 3, Gate 4, Gate 10, Gate 11, Gate 14, Gate 15 and
Gate 17). We labeled these flights as F1, F2, F3, F4, F5, F6 and F7. These flights needed to be assigned to gates based on the considerations of walking distance for passengers between the flights and baggage transport distance between gates.

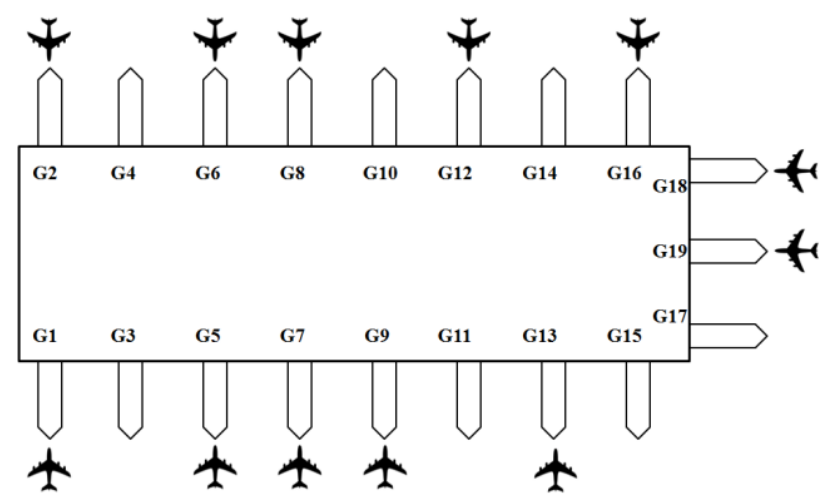

Figure 1. C Concourse at San Francisco Airport with 12 aircrafts will be departed.

The number of the passenger who attach to the other departing gate for these flights is shown in Table 1. As an example, there are 5 passengers from flight 1 (F1) must walk to the gate 1 from their next departure. Meanwhile, Table 2 presents the distance (in yards) between the arrival gates and the next departure gates.

Table .1 Passenger flow in the terminal

\begin{tabular}{cccccccccccccccccccc}
\hline \multirow{2}{*}{ Flight } & $\mathbf{1}$ & $\mathbf{2}$ & $\mathbf{3}$ & $\mathbf{4}$ & $\mathbf{5}$ & $\mathbf{6}$ & $\mathbf{7}$ & $\mathbf{8}$ & $\mathbf{9}$ & $\mathbf{1 0}$ & $\mathbf{1 1}$ & $\mathbf{1 2}$ & $\mathbf{1 3}$ & $\mathbf{1 4}$ & $\mathbf{1 5}$ & $\mathbf{1 6}$ & $\mathbf{1 7}$ & $\mathbf{1 8}$ & $\mathbf{1 9}$ \\
\hline F1 & 5 & 5 & 10 & 8 & 15 & 8 & 2 & 10 & 8 & 20 & 5 & 4 & 0 & 9 & 3 & 4 & 1 & 2 & 1 \\
F2 & 5 & 2 & 1 & 4 & 19 & 9 & 4 & 2 & 3 & 2 & 27 & 3 & 8 & 4 & 0 & 2 & 1 & 7 & 2 \\
F3 & 10 & 0 & 4 & 9 & 13 & 4 & 4 & 4 & 3 & 5 & 5 & 8 & 4 & 9 & 11 & 7 & 9 & 4 & 4 \\
F4 & 4 & 8 & 5 & 4 & 10 & 4 & 1 & 0 & 0 & 2 & 4 & 19 & 1 & 2 & 4 & 5 & 5 & 8 & 2 \\
F5 & 4 & 11 & 9 & 9 & 6 & 3 & 1 & 4 & 4 & 2 & 1 & 0 & 3 & 5 & 1 & 2 & 2 & 3 & 4 \\
F6 & 1 & 2 & 42 & 5 & 2 & 7 & 6 & 2 & 4 & 7 & 2 & 3 & 6 & 4 & 10 & 2 & 1 & 0 & 0 \\
F7 & 3 & 3 & 2 & 5 & 9 & 13 & 11 & 2 & 2 & 3 & 7 & 22 & 4 & 0 & 1 & 1 & 2 & 2 & 9 \\
\hline
\end{tabular}

Table.2 Distance matrix in the terminal (yards)

\begin{tabular}{cccccccccccccccccccc}
\hline \multirow{2}{*}{ Gate } & $\mathbf{1}$ & $\mathbf{2}$ & $\mathbf{3}$ & $\mathbf{4}$ & $\mathbf{5}$ & $\mathbf{6}$ & $\mathbf{7}$ & $\mathbf{8}$ & $\mathbf{9}$ & $\mathbf{1 0}$ & $\mathbf{1 1}$ & $\mathbf{1 2}$ & $\mathbf{1 3}$ & $\mathbf{1 4}$ & $\mathbf{1 5}$ & $\mathbf{1 6}$ & $\mathbf{1 7}$ & $\mathbf{1 8}$ & $\mathbf{1 9}$ \\
\hline 3 & 10 & 40 & - & 30 & 10 & 40 & 20 & 50 & 30 & 60 & 40 & 70 & 50 & 80 & 60 & 90 & 70 & 90 & 80 \\
4 & 40 & 10 & 30 & - & 40 & 10 & 50 & 20 & 60 & 30 & 70 & 40 & 80 & 50 & 90 & 60 & 90 & 70 & 80 \\
10 & 70 & 40 & 60 & 30 & 50 & 20 & 40 & 10 & 30 & - & 40 & 10 & 50 & 40 & 60 & 30 & 70 & 40 & 50 \\
11 & 50 & 80 & 40 & 70 & 30 & 60 & 20 & 50 & 10 & 40 & - & 30 & 10 & 40 & 20 & 50 & 30 & 50 & 40 \\
14 & 90 & 60 & 80 & 50 & 70 & 40 & 60 & 30 & 50 & 20 & 40 & 10 & 30 & - & 40 & 10 & 50 & 20 & 30 \\
15 & 70 & 100 & 60 & 90 & 50 & 80 & 40 & 70 & 30 & 60 & 20 & 50 & 10 & 40 & - & 30 & 10 & 30 & 20 \\
17 & 80 & 100 & 70 & 90 & 60 & 80 & 50 & 70 & 40 & 60 & 30 & 50 & 20 & 40 & 10 & 30 & - & 20 & 10 \\
\hline
\end{tabular}

In another hand, the amount of transit bags, mail, and cargo from each of the arriving flights to departing gates is shown in Table 4. Then, Table 6 shows the distance of the trailers on the ramp. 
Table. 4 Baggage flow (units of baggage)

\begin{tabular}{cccccccccccccccccccc}
\hline Flight & $\mathbf{1}$ & $\mathbf{2}$ & $\mathbf{3}$ & $\mathbf{4}$ & $\mathbf{5}$ & $\mathbf{6}$ & $\mathbf{7}$ & $\mathbf{8}$ & $\mathbf{9}$ & $\mathbf{1 0}$ & $\mathbf{1 1}$ & $\mathbf{1 2}$ & $\mathbf{1 3}$ & $\mathbf{1 4}$ & $\mathbf{1 5}$ & $\mathbf{1 6}$ & $\mathbf{1 7}$ & $\mathbf{1 8}$ & $\mathbf{1 9}$ \\
\hline F1 & 19 & 28 & 11 & 8 & 30 & 25 & 33 & 5 & 49 & 14 & 38 & 38 & 14 & 23 & 17 & 4 & 20 & 44 & 8 \\
F2 & 43 & 40 & 22 & 29 & 4 & 49 & 8 & 6 & 20 & 21 & 17 & 5 & 27 & 29 & 29 & 40 & 42 & 34 & 25 \\
F3 & 22 & 17 & 36 & 45 & 22 & 28 & 17 & 23 & 18 & 44 & 12 & 8 & 41 & 48 & 25 & 11 & 27 & 47 & 28 \\
F4 & 47 & 11 & 4 & 26 & 16 & 21 & 24 & 8 & 45 & 22 & 45 & 20 & 14 & 22 & 32 & 32 & 9 & 39 & 7 \\
F5 & 3 & 24 & 46 & 38 & 48 & 7 & 24 & 33 & 29 & 43 & 7 & 21 & 45 & 47 & 28 & 11 & 17 & 3 & 23 \\
F6 & 9 & 47 & 18 & 3 & 44 & 14 & 4 & 27 & 34 & 38 & 17 & 26 & 2 & 3 & 28 & 40 & 11 & 8 & 46 \\
F7 & 46 & 34 & 48 & 42 & 26 & 12 & 40 & 49 & 18 & 36 & 24 & 6 & 18 & 9 & 2 & 10 & 14 & 47 & 9 \\
\hline
\end{tabular}

Table.6 Distance matrix for baggage trailers on the ramp (yards)

\begin{tabular}{|c|c|c|c|c|c|c|c|c|c|c|c|c|c|c|c|c|c|c|c|}
\hline \multirow{2}{*}{ Gate } & \multicolumn{19}{|c|}{ Departing Gate } \\
\hline & 1 & 2 & 3 & 4 & 5 & 6 & 7 & 8 & 9 & 10 & 11 & 12 & 13 & 14 & 15 & 16 & 17 & 18 & 19 \\
\hline F1 & 15 & 60 & - & 45 & 15 & 60 & 30 & 75 & 45 & 90 & 60 & 105 & 75 & 120 & 90 & 135 & 105 & 135 & 120 \\
\hline $\mathrm{F} 2$ & 68 & 17 & 51 & - & 68 & 17 & 85 & 34 & 102 & 51 & 119 & 68 & 136 & 85 & 153 & 102 & 153 & 119 & 136 \\
\hline F3 & 112 & 64 & 96 & 48 & 80 & 32 & 64 & 16 & 48 & - & 64 & 16 & 80 & 64 & 96 & 48 & 112 & 64 & 80 \\
\hline $\mathrm{F} 4$ & 65 & 104 & 52 & 91 & 39 & 78 & 26 & 65 & 13 & 52 & - & 39 & 13 & 52 & 26 & 65 & 39 & 65 & 52 \\
\hline F5 & 135 & 90 & 120 & 75 & 105 & 60 & 90 & 45 & 75 & 30 & 60 & 15 & 45 & - & 60 & 15 & 75 & 30 & 45 \\
\hline F6 & 98 & 140 & 84 & 126 & 70 & 112 & 56 & 98 & 42 & 84 & 28 & 70 & 14 & 56 & - & 42 & 14 & 42 & 28 \\
\hline F7 & 112 & 140 & 98 & 126 & 84 & 112 & 710 & 98 & 56 & 84 & 42 & 70 & 28 & 56 & 14 & 42 & - & 28 & 14 \\
\hline
\end{tabular}

\section{EXPERIMENTAL RESULTS AND ANALYSIS}

We calculated the matrix multiplication by using Table 1 and Table 2. Then, we obtained the result as Table 3 which is the total walking distances of the passenger.
Normally, the baggage is transport by the trailers from one gate to another gate on the ramp. We assume the capacity of 5 bags for each trailer. Therefore from the assumption and the data in Table 4 , we obtained the results of Table 5.

Table.3 Travelling distance (yards)

\begin{tabular}{cccccccc}
\hline Flight & $\mathbf{9}$ & $\mathbf{1 0}$ & $\mathbf{1 1}$ & $\mathbf{1 4}$ & $\mathbf{1 5}$ & $\mathbf{1 7}$ \\
\hline F1 & 5,010 & 4,390 & 3,820 & 4,870 & 5,060 & 6,650 & 7,090 \\
F2 & 4,240 & 5,290 & 4,190 & 3,020 & 4,650 & 4,400 & 4,970 \\
F3 & 5,610 & 5,950 & 4,930 & 4,270 & 4,910 & 4,950 & 5,320 \\
F4 & 4,500 & 3,990 & 3,280 & 3,580 & 3,460 & 4,320 & 4,460 \\
F5 & 2,950 & 2,720 & 3,060 & 3,490 & 3,620 & 4,330 & 4,530 \\
F6 & 3,060 & 4,310 & 4,740 & 3,900 & 5,760 & 5,300 & 6,020 \\
F7 & 4,680 & 4,380 & 3,290 & 3,620 & 3,970 & 4,960 & 5,220 \\
\hline
\end{tabular}


Table.5 Baggage flow in number of trips for trailers

\begin{tabular}{cccccccccccccccccccc}
\hline \multirow{2}{*}{ Flight } & $\mathbf{1}$ & $\mathbf{2}$ & $\mathbf{3}$ & $\mathbf{4}$ & $\mathbf{5}$ & $\mathbf{6}$ & $\mathbf{7}$ & $\mathbf{8}$ & $\mathbf{9}$ & $\mathbf{1 0}$ & $\mathbf{1 1}$ & $\mathbf{1 2}$ & $\mathbf{1 3}$ & $\mathbf{1 4}$ & $\mathbf{1 5}$ & $\mathbf{1 6}$ & $\mathbf{1 7}$ & $\mathbf{1 8}$ & $\mathbf{1 9}$ \\
\hline F1 & 4 & 6 & 3 & 2 & 6 & 5 & 7 & 1 & 10 & 3 & 8 & 8 & 3 & 5 & 4 & 1 & 4 & 9 & 2 \\
F2 & 9 & 8 & 5 & 6 & 1 & 10 & 2 & 2 & 4 & 5 & 4 & 1 & 6 & 6 & 6 & 8 & 9 & 7 & 5 \\
F3 & 5 & 4 & 8 & 9 & 5 & 6 & 4 & 5 & 4 & 9 & 3 & 2 & 9 & 10 & 5 & 3 & 6 & 10 & 6 \\
F4 & 10 & 3 & 1 & 6 & 4 & 5 & 5 & 2 & 9 & 5 & 9 & 4 & 3 & 5 & 7 & 7 & 2 & 8 & 2 \\
F5 & 1 & 5 & 10 & 8 & 10 & 2 & 5 & 7 & 6 & 9 & 2 & 5 & 9 & 10 & 6 & 3 & 4 & 1 & 5 \\
F6 & 2 & 10 & 4 & 1 & 9 & 3 & 1 & 6 & 7 & 8 & 4 & 6 & 1 & 1 & 6 & 8 & 3 & 2 & 10 \\
F7 & 10 & 7 & 10 & 9 & 6 & 3 & 8 & 10 & 4 & 8 & 5 & 2 & 4 & 2 & 1 & 2 & 3 & 10 & 2 \\
\hline
\end{tabular}

Furthermore, we obtained the result as Table 7 by using Table 5 and Table 6 . The table below calculates by using matrix multiplication. Therefore, Table 7 shows the total baggage transport distances.

Table.7 Baggage transport distance (yards)

\begin{tabular}{cccccccc}
\hline Flight & $\mathbf{7}$ & $\mathbf{1 0}$ & $\mathbf{1 1}$ & $\mathbf{1 4}$ & $\mathbf{1 5}$ & $\mathbf{1 7}$ \\
\hline F1 & 6,420 & 7,820 & 5,616 & 4,004 & 5,685 & 5,516 & 5,936 \\
F2 & 7,965 & 8,636 & 6,992 & 5,707 & 6,525 & 6,762 & 6,986 \\
F3 & 8,460 & 9,197 & 7,136 & 5,824 & 6,690 & 7,224 & 7,518 \\
F4 & 7,005 & 8,296 & 6,064 & 4,537 & 6,015 & 5,922 & 6,426 \\
F5 & 7,320 & 8,398 & 6,560 & 5,174 & 6,600 & 7,000 & 7,546 \\
F6 & 6,975 & 7,480 & 5,328 & 4,719 & 5,565 & 5,978 & 6,244 \\
F7 & 6,450 & 7,327 & 6,528 & 5,772 & 7,590 & 8,008 & 8,470 \\
\hline
\end{tabular}

We now present our results of the ILP model. We implemented the zero-one integer problem and B\&B that ran with AIMMS Software for CPLEX. There are three different pair of weight for the total distance of the passenger flow in the terminal $\left(w_{1}\right)$ and of the distance for the baggage transport $\left(w_{2}\right):\left\{w_{1}, w_{2}\right\}=\{3,7\},\{5,5\}$ and $\{7,3\}$,

\section{Case 1: $\left\{w_{1}, w_{2}\right\}=\{3,7\}$}

From Table 8, we can view that F1 was assigned to the gate $11, \mathrm{~F} 2$ assigned to gate $17, \mathrm{~F} 3$ assigned to gate $14, \mathrm{~F} 4$ assigned to gate $15, \mathrm{~F} 5$ assigned to gate $3, \mathrm{~F} 6$ assigned to gate 10 , and F7 assigned to gate 4 . With these result, we can calculate the optimal solution with minimize total distance of 398,459 yards.

Table. 8 The result of the flight assigned to the gate with the ratio of $3: 7$

\begin{tabular}{cc}
\hline Flight & Gate assigned to \\
\hline F1 & 11 \\
F2 & 17 \\
F3 & 14 \\
F4 & 15 \\
F5 & 3 \\
F6 & 10 \\
F7 & 4 \\
\hline
\end{tabular}

\section{Case 2: $\left\{w_{1}, w_{2}\right\}=\{5,5\}$}

We can observe the result in Table 9 that F1 was assigned to the gate 10, F2 assigned to gate 11, F3 assigned to gate $15, \mathrm{~F} 4$ assigned to gate $17, \mathrm{~F} 5$ assigned to gate 14 , F6 assigned to gate 3 , and F7 assigned to gate 4 . Then, we minimized the total distance with the optimal solution of 365,925 yards.

Table.9 The result of the flight assigned to the gate with the ratio of $5: 5$

\begin{tabular}{cc}
\hline Flight & Gate assigned to \\
\hline F1 & 10 \\
F2 & 11 \\
F3 & 15 \\
F4 & 17 \\
F5 & 14 \\
F6 & 3 \\
F7 & 4 \\
\hline
\end{tabular}

\section{Case 3: $\left\{w_{1}, w_{2}\right\}=\{7,3\}$}

The last test of the ratio for the both weighted of $7: 3$, we obtained the result as Table 10 shows that $F 1$ is assigning to the gate $10, \mathrm{~F} 2$ assigned to gate $11, \mathrm{~F} 3$ assigned to gate 15, F4 assigned to gate 17, F5 assigned to 
gate 4, F6 assigned to gate 3, and F7 assigned to gate 14 . We obtained the total distance of 325,808 yards which is the optimal solution for this case.

Table.10 The result of the flight assigned to the gate with the ratio of $7: 3$

\begin{tabular}{cc}
\hline Flight & Gate assigned to \\
\hline F1 & 10 \\
F2 & 11 \\
F3 & 15 \\
F4 & 17 \\
F5 & 4 \\
F6 & 3 \\
F7 & 14 \\
\hline
\end{tabular}

Based on these three cases, we observed that the outcome of the flights that assigned to the gates is different. The different outcomes will produce the different optimal solution for each case that tested. Therefore, we make conclude that the results of the flights that assigned to gates is strongly linked to the ratio of the both weighted.

\section{CONCLUSION}

In this paper, we proposed an ILP model to assist airline's operation in gate assignment. We formulated the model as zero-one integer problem to obtain the optimization. We solved the problem using AIMMS Software for CPLEX. In the software, it can generate the branch-and-bound method that can efficiency to solve it.

From the results that we obtained from the three cases that been tested, we can view that there has the different between the gate assigned for the same flight within these three cases. Meanwhile, these conditions occurred with the reason of the ratio of the weighted between the total walking distance and the total distance of the baggage transport. Therefore, the ratio for the both weighted can affected for assigning the flight to the gate.

Although the results based on the ratio of the weighted for both criterion, but it can efficiency to assigning the flights. On the others hand, the time of the flight arrival and departure will affect the results for assigning the flights is more challenging in the future study..

\section{ACKNOWLEDGEMENTS}

The authors would like to thank especially to MOE, Universiti Teknologi Malaysia (UTM) and GUP (the vote number $08 \mathrm{H} 49$ ) for funding the project.

\section{REFERENCES}

[1] Bazargan, M. Airline Operations and Scheduling, USA: Ashgate Publishing Limited, 2010, pp. 171-182.

[2] Pinedo, M. Scheduling Theory, Algorithms, and Systems, New York: Prentice Hall, 2008, pp. 2-3.

[3] Ding, H.; Lim, A.; Rodrigues B.; Zhu, Y. Computers \& Operations Research 32 (2004) 1867-1880.

[4] Yan, S.Y.; Huo, C.-M. Transpotation Research 35(5) (1999) 413 432.

[5] Gregory, D.; Phillip, J. L. Transportation Science 27(3) (1993) 281297

[6] Michael, W. C.; Camille, C. P. Operations Research - A Practical Introduction, USA: CRC Press LLC, 2001, pp. 1-416.

[7] Paul, E. F. Linear And Nonlinear Programming With Maple. USA: CRC Press, 2010, pp. 145-157. 